\title{
$\epsilon$ Subunit-Containing Acetylcholine Receptors in Myotubes Belong to the Slowly Degrading Population
}

\author{
Carlo Sala, ${ }^{1}$ James O'Malley, ${ }^{2}$ Rufeng Xu, ${ }^{2}$ Guido Fumagalli, ${ }^{3}$ and Miriam M. Salpeter ${ }^{2}$ \\ ${ }^{1}$ Consiglio Nazionale delle Ricerche, Center of Cellular and Molecular Pharmacology, Department of Medical \\ Pharmacology, University of Milan, 20129 Milan, Italy, ${ }^{2}$ Department of Neurobiology and Behavior, Cornell University, \\ Ithaca, New York 14853, and 3/nstitute of Pharmacology, School of Medicine, University of Verona, 37134 Verona, Italy
}

Two types of muscle acetylcholine receptors (AChRs) can be distinguished on the basis of their degradation rates and sensitivities to innervation, muscle activity, and agents elevating intracellular cAMP. The first type (Rs), is present in a stable form (degradation $t_{1 / 2}=\sim 10 \mathrm{~d}$ ) at the adult innervated neuromuscular junctions (NMJs). Rs can also exist in a less stable form (called accelerated Rs; $t_{1 / 2}=\sim 3-5 \mathrm{~d}$ ) at denervated NMJs and in aneurally cultured myotubes; agents that increase intracellular cAMP reversibly modulate Rs stability. The second type of $A C h R$ is a rapidly degrading receptor $(\mathrm{Rr})$ expressed only in embryonic and noninnervated muscles. $\mathrm{Rr}$ can be stabilized by ATP and not by cAMP. This study tested the hypothesis that the degradation properties unique to the Rs are attributable to the presence of the $\epsilon$ subunit.
Immunoprecipitation and Western blot analysis of AChRs extracted from rat muscle cells in tissue culture showed that AChRs recognized by antibodies against the $\epsilon$ subunit degraded as a single population with a half-life similar to that of the slow component, Rs, in these cells. In addition, as for Rs receptors in denervated NMJs and cultured muscle cell, the degradation rate of these $\epsilon$-containing AChRs was stabilized by dibutyryl-cAMP. The data indicate that the $\epsilon$-containing AChRs behave like Rs. Thus, the presence of the $\epsilon$ subunit is sufficient for selecting an AChR molecule to the Rs pool.

Key words: AChR degradation; AChR subunit; myotubes; neuromuscular junction; immunoprecipitation; monoclonal antibodies; $\alpha$-bungarotoxin; cAMP
The turnover of neurotransmitter receptors at the postsynaptic membrane may influence the stability of synaptic contacts and the ability of the cell to modify rapidly the number and the properties of individual synaptic boutons (Rich and Lichtman, 1989). Yet knowledge of the mechanism underlying this regulation is essentially unknown, and what little is know is limited to the muscle acetylcholine receptor (AChR). However, information now emerging on that regulation at the neuromuscular junction may help clarify the overall mechanism and pioneer studies of events occurring also at neuronal synapses.

Two distinct metabolic forms of muscle AChRs have been identified based on their degradation characteristics (Michler and Sakmann, 1980; Levitt and Salpeter, 1981; Stanley and Drachman, 1981; Shyng and Salpeter, 1989, 1990). Slowly degrading receptors $\left(\mathrm{Rs} ; t_{1 / 2}=10 \mathrm{~d}\right)$ are primarily expressed at adult innervated neuromuscular junctions (NMJs), whereas most of the AChRs expressed by embryonic and denervated adult muscles are rapidly degrading $\left(\mathrm{Rr} ; t_{1 / 2}=1-2 \mathrm{~d}\right)$. The muscle AChR has a subunit stoichiometry of $\alpha_{2} \beta \delta \gamma / \epsilon$ (Karlin, 1993; Duclert and Changeaux, 1995), and the mutually exclusive $\gamma$ and $\epsilon$ subunits endow the receptors with different electrophysiological properties

Received Aug. 26, 1997; accepted Sept. 12, 1997.

This work is supported by NATO Grant CGR.960655, Telethon Italy Grant 764 Biomed Grant Project CT-931100 to G.F., and National Institutes of Health Grant NS09315 to M.M.S. C.S. is supported by Telethon Italy (Dottorato in Farmacologia e Tossicologia, University of Milan). We thank Profs. S. Tzartos (Pasteur Institute Hellenique, Athens, Greece) and S. C. Froehner (University of North Carolina, Chapel Hill, NC) for the gift of some of the mAbs used in this study, Dr. Steven Heinemann (Salk Institute, La Jolla, CA) for the gift of AChR subunit constructs, Rui Lin for help in characterizing the 52Abe antibody, Profs N. Borgese and F. Clementi for valuable support and critical reading of this manuscript, and Dr. Joel Stiles for help in computer image processing.

Correspondence should be addressed to Prof. Guido Fumagalli, Institute of Pharmacology, School of Medicine, Ospedale Policlinico Borgo Roma, 37134 Verona, Italy.

Copyright (C) 1997 Society for Neuroscience $\quad 0270-6474 / 97 / 178937-08 \$ 05.00 / 0$
(Mishina et al., 1986; Naranjo and Brehm, 1993). Whether subunit composition also endows the receptors with different metabolic properties is the open question addressed by this study.

Both $\epsilon$-AChRs and Rs predominate in the adult innervated NMJ (Levitt and Salpeter, 1981; Gu and Hall, 1988); both $\gamma$ AChRs and Rr appear in adult muscle after denervation and are downregulated by electrical stimulation of denervated muscles (Goldman et al., 1988; Fumagalli et al., 1990; Witzemann et al., 1991). Finally, when Rr and Rs coexist at the NMJ during interim periods when the two AChR populations are replacing each other (Shyng and Salpeter, 1990), both $\gamma$ - and $\epsilon$-AChRs are also present. (Vicini and Schuetze, 1985; Gu and Hall, 1988; Missias et al., 1996).

These correlations suggest that subunit composition may confer AChR metabolic properties. Yet, measurements of $\epsilon$ - or $\gamma$-AChR degradation in heterologous (nonmuscle) expression systems have provided conflicting results (Gu et al., 1990; Jayawickreme and Claudio, 1994; Kopta and Steinbach, 1994; Liu et al., 1994). Furthermore, neither the changes in $\epsilon$ subunit expression nor the changes in AChR channel properties (a clear indication of subunit switch) are coincident with metabolic stabilization during NMJ maturation (for review, see Hall and Sanes, 1993).

Some of the discrepancies may be attributable to the fact that degradation rate alone is an insufficient criterion for characterizing Rs and Rr and must be combined with response to stabilizing factors before an unequivocal identification can be made. Both $\mathrm{Rr}$ and Rs can have intermediate and sometimes overlapping $t_{1 / 2}$ values, yet each has unique stabilizing responses (Shyng et al., 1991; O'Malley et al., 1993, 1997).

In this study we used immunoprecipitation and Western blot analysis with anti- $\epsilon$ subunit antibodies to measure the degradation rate of $\epsilon$-AChR. We found that the $\epsilon$-containing AChRs are exclusively of the Rs, cAMP-sensitive population. The relation- 
ship between degradation characteristics and subunit composition will be discussed.

\section{MATERIALS AND METHODS}

\section{Muscle cell culture}

Muscle cell cultures were prepared as published previously (O’Malley et al., 1993, 1996). Myoblasts were isolated from the hindlimb muscles of embryonic day 18-19 Sprague Dawley rat embryos by $0.05 \%$ type $1 \mathrm{~A}$ collagenase (Sigma, St Louis MO) digestion in DMEM for $3 \mathrm{hr}$ at $37^{\circ} \mathrm{C}$. The mononucleated cells were separated from tissue debris by filtration and plated in 35 or $100 \mathrm{~mm}$ tissue culture dishes coated with $0.7 \mathrm{mg} / \mathrm{cm}^{2}$ Matrigel (Becton Dickinson Labware, Bedford, MA) at a density of $5 \times$ $10^{5}$ cells $/ \mathrm{cm}^{2}$. The myoblasts were grown to confluence $(2-3 \mathrm{~d})$ in DMEM supplemented with $20 \%$ fetal calf serum and then in DMEM supplemented with $10 \%$ horse serum and maintained at $37^{\circ} \mathrm{C}$ in a humidified atmosphere of $90 \%$ air $/ 10 \% \mathrm{CO}_{2}$.

\section{Labeling of $A C h R s$}

AChRs from cultured muscle cells or from innervated and denervated soleus muscles were labeled with radioactive $\alpha$-bungarotoxin ( ${ }^{125} \mathrm{I}$-BTX; Amersham, Buckinghamshire, UK; specific activity, $>200 \mathrm{Ci} / \mathrm{mmol})$. Cultured muscle cells were incubated with $20 \mathrm{nM}^{125}$ I-BTX in Dulbecco's PBS (D-PBS) containing $0.1 \%$ bovine serum albumin (BSA) for $1 \mathrm{hr}$ at room temperature. The unbound toxin was removed by three washes (5 min each) with $0.1 \%$ BSA in D-PBS. The innervated or 1 week denervated adult rat soleus muscles were incubated with $20 \mathrm{nM}{ }^{125} \mathrm{I}-\mathrm{BTX}$ for 2 $\mathrm{hr}$ at room temperature in continuously oxygenated D-PBS containing $0.1 \%$ BSA. In both cases, nonspecific binding was determined by preincubating with a 100-fold excess of nonradioactive BTX (Sigma) before the addition of ${ }^{125}$ I-BTX.

\section{AChR extraction}

For Western blot analysis. AChRs were extracted from cultured myotubes with Triton X-100. All procedures were performed at $4^{\circ} \mathrm{C}$. The media were removed from three $100 \mathrm{~mm}$ plastic tissue culture dishes, and the dishes were rinsed three times with a homogenization buffer [in mM: 100 $\mathrm{NaCl}, 1 \mathrm{NaN}_{3}, 0.1$ phenylmethylsulfonyl fluoride (PMSF), 1 EDTA, 1 EGTA, and 20 Tris, $\mathrm{pH}$ 7.2]. Cells were scraped from the plates with a rubber policeman in $5 \mathrm{ml}$ of homogenization buffer, pooled, and homogenized with a glass tissue homogenizer. The combined suspension was centrifuged for $10 \mathrm{~min}$ at $10,000 \mathrm{rpm}$ in a JA $20 \cdot 1$ rotor (Sorvall, Wilmington, Germany), and the supernatant was discarded. The pellet was homogenized in $1 \mathrm{ml}$ of detergent buffer (homogenization buffer and $1 \%$ Triton $\mathrm{X}-100$ ) and incubated, while rotating, for $2 \mathrm{hr}$ in an Eppendorf (Hamburg, Germany) tube. The suspension was then centrifuged for $30 \mathrm{~min}$ at 10,000 $\mathrm{rpm}$ in a JA $20 \cdot 1$ rotor, and the pellet was discarded. The supernatant was further processed for Western blot analysis as described below.

For immunoprecipitation assay. AChRs from both tissue-cultured and adult muscles were extracted for this assay. The cultured cells were scraped from $100 \mathrm{~mm}$ plates using a rubber policeman in $1 \mathrm{ml}$ of D-PBS containing $2 \mathrm{~mm}$ EDTA, $2 \mathrm{~mm}$ PMSF, $2 \mu \mathrm{g} / \mathrm{ml}$ leupeptin, $2 \mu \mathrm{g} / \mathrm{ml}$ aprotinin, and $2 \mu \mathrm{g} / \mathrm{ml}$ pepstatin. The suspension was homogenized in a glass tissue homogenizer, and Triton $\mathrm{X}-100$ was added to a final concentration of $1 \%$. After $2 \mathrm{hr}$ of extraction on a rotating shaker at $4^{\circ} \mathrm{C}$, the suspension was centrifuged for $1 \mathrm{hr}$ at $10,000 \mathrm{rpm}$ in a refrigerated Eppendorf 5417R microfuge at $4^{\circ} \mathrm{C}$, and the supernatant was collected.

Innervated or 1 week denervated adult rat muscle was homogenized in a Ultraturrex blender in 4 volumes of an ice-cold homogenization buffer (in mM: 50 phosphate buffer, $\mathrm{pH} 7.4,50 \mathrm{NaCl}, 2$ EDTA, 2 EGTA, and 2 PMSF). The homogenate was centrifuged for $30 \mathrm{~min}$ at $12,000 \mathrm{rpm}$ in a GSA rotor (Sorvall), and the pellet was resuspended in 4 volumes of the homogenization buffer. After centrifugation, the pellet was dissolved in 1 volume of an extraction buffer (50 mM phosphate buffer, $\mathrm{pH} 7.4,1 \mathrm{M}$ $\mathrm{NaCl}, 2$ mM EDTA, 2 mm EGTA, and 2 mM PMSF), and Triton X-100 was added to a final concentration of $2 \%$. After $2 \mathrm{hr}$ of extraction at $4^{\circ} \mathrm{C}$ with mild stirring, the AChR-containing supernatant was clarified by centrifugation for $2 \mathrm{hr}$ at $12,000 \mathrm{rpm}$ in a GSA rotor.

\section{Estimation of AChR degradation}

Degradation curves of total surface AChRs or of specific $\epsilon$ subunitcontaining AChRs were performed using three methods: (1) by release of radioactivity after labeling cells with ${ }^{125} \mathrm{I}-\alpha$-BTX as described previously (O'Malley et al., 1993); (2) by immunoprecipitation of labeled AChRs using either anti- $\alpha$ or anti- $\epsilon$ subunit-specific antibodies; and (3) by
Western blot analysis to identify $\epsilon$ subunits from AChRs extracted at different times after labeling, using a new anti- $\epsilon$ subunit antibody (52Ab $\epsilon$ ).

For determining degradation by the standard radioactive release method. AChRs were labeled as indicated above. At daily intervals up to $15 \mathrm{~d}$ after labeling the medium was removed, released radioactivity was counted in a gamma counter, and fresh medium was placed on the cells. At the end of the experiment, cells were scraped off the dish, and all remaining radioactivity was counted and added to the sum of the released activity. The total gave a value for label on day 0 . The half-lives and relative contents of the fast (Rr) and slow (Rs) components were determined from their slopes and $y$-intercepts on a degradation curve as described previously (O'Malley et al., 1993). The observed degradation values were corrected for ${ }^{125} \mathrm{I}$ decay. No correction was made for BTX unbinding, because half-life values in the literature are uncertain. Cohen et al. (1990) give a value of $56 \mathrm{~d}$, which is lower than preliminary results seen by us (data not shown). However, because the unbinding values are long compared with the degradation half-lives in this study, any such correction would make little difference to the reported results.

For determining degradation by immunoprecipitation. Assays were performed on AChRs extracted from cultured muscle cells as well as from normal and denervated adult muscle. The subunit-specific immunoprecipitation of AChR was performed as described by Green and Claudio (1993). Briefly, at different times after labeling, $100 \mu \mathrm{l}$ aliquots of extracts from ${ }^{125}$ I-BTX-labeled myotube or muscle tissue were first counted to obtain total radioactivity and then incubated with subunit-specific antibodies. Triplicate samples of the same extract were used for each antibody. The subunit-specific rat anti- $\alpha$ mAb 155 and anti- $\epsilon$ mAb 168 (Tzartos et al., 1986; Engel et al., 1993) were routinely used. After overnight incubation of $100 \mu \mathrm{l}$ of extract with $0-6 \mu \mathrm{l}$ of primary antibody at $4^{\circ} \mathrm{C}$ in a rotating shaker, $10 \mu \mathrm{l}$ of $50 \%$ diluted protein $\mathrm{G}-$ Sepharose (Sigma) was added for a $90 \mathrm{~min}$ incubation at $4^{\circ} \mathrm{C}$ in a rotating shaker to precipitate rat $\mathrm{IgG}$. The immunocomplex was then washed three times with $1 \mathrm{ml}$ of PBS containing $1 \mathrm{~mm}$ EDTA, $2 \mathrm{mM}$ PMSF, $2 \mu \mathrm{g} / \mathrm{ml}$ leupeptin, $2 \mu \mathrm{g} / \mathrm{ml}$ aprotinin, and $2 \mu \mathrm{g} / \mathrm{ml}$ pepstatin, and the immunoprecipitated radioactivity was counted in a gamma counter and compared with the total radioactivity of the extract. The data at the various time points were expressed as the fraction of the total radioactivity that was immunoprecipitated by each $\mathrm{mAb}$ relative to that fraction at the time of ${ }^{125}$ I-BTX labeling.

For determining degradation by Western blot analysis. The measurements require that only the receptor molecules present at the day of labeling with ${ }^{125}$ I-BTX (degradation day 0) are loaded for SDS-PAGE. This was done by separating the labeled AChR from unlabled receptor synthesized after the day of labeling. After extracting AChRs from tissue culture dishes as described above, all free $\alpha$-BTX binding sites were removed by incubating the supernatant $(1 \mathrm{ml})$ overnight at $4^{\circ} \mathrm{C}$ with a 500 $\mu \mathrm{l}$ suspension of $\alpha$-BTX-Sepharose-conjugated beads prepared as described by Gotti et al. (1982). The next day the beads with bound AChRs were pelleted with a pulse centrifugation in a microcentrifuge and discarded. Because the AChRs labeled with ${ }^{125}$ I-BTX would not bind to the beads, only unlabeled receptors would have bound and thus would be removed from the mixture. Confirmation that all unlabeled AChRs had been removed by this method was achieved by relabeling a small aliquot of the supernatant and determining that there were no remaining free BTX binding sites. Once it was determined that the only AChRs in the supernatant were those labeled on degradation day 0 , the supernatant was centrifuged in concentrating tubes with a molecular weight cutoff of $5 \mathrm{kDa}$ (Millipore, Bedford, MA) to a volume of $50 \mu \mathrm{l}$. This sample was added to $50 \mu \mathrm{l}$ of $2 \times$ sample buffer (125 mM Tris, $20 \%$ glycerol, $2 \%$ 2-mercaptoethanol, $2 \% \mathrm{SDS}$, and $40 \mu \mathrm{g} / \mathrm{ml}$ bromophenol blue, $\mathrm{pH} 6.8$ ) to a final volume of $100 \mu \mathrm{l}$ and boiled for $10 \mathrm{~min}$. To remove any bias associated with potential variance in extraction and purification, the relative radioactive contents from samples on days $1,2,4,7$, and 10 were compared with those seen on the parallel standard degradation curve (obtained by the standard radioactive release method above) in which no extraction was involved. The final volumes were then adjusted such that the total radioactivity per microliter for each day relative to that on degradation day 0 was the same as for the standard degradation curve. Because the correction included total AChRs, it did not bias any determination of the relative amount of $\epsilon$-containing $\mathrm{AChR}$ present in each sample. To achieve the same ionic balance in each extract, a $50 \mu \mathrm{l}$ sample from each AChR extract was then microdialyzed for $2 \mathrm{hr}$ at room temperature against sample buffer using a membrane with a $7 \mathrm{kDa}$ molecular weight cutoff. The remainder of the extracts from each day was pooled and diluted 1:1, 1:2,1:4, and 1:8 with sample buffer and, $50 \mu \mathrm{l}$ of 
each diluent was also microdialyzed. This serial dilution was used to determine linearity in the Western analysis.

\section{Western blot analysis}

Samples were run on either an $8.0 \%$ or an $8.5 \%$ polyacrylamide gel and then transferred to a polyvinylidene difluoride (PVDF) membrane. For verification of specificity of the new anti- $\epsilon$ subunit antibody, $52 \mathrm{Ab} \epsilon$, membrane preparations from HEK 293 cells, transfected with different combinations of AChR subunits (see below), were used. The PVDF membrane was washed with Tris buffer $(50 \mathrm{~mm}$, pH 7.2$)$ with $0.1 \%$ Tween 20 and then incubated with either anti- $\epsilon$ (52Ab $\epsilon, 1: 2000)$ or anti- $\gamma / \delta$ (88B, 1:1000) (Froehner et al., 1983) in Tris buffer, pH 7.2, with $0.1 \%$ Tween 20 and $4.5 \%$ dry milk overnight at $4^{\circ} \mathrm{C}$. The membrane was washed twice followed by incubation with peroxidase-conjugated antirabbit $\operatorname{IgG}$ for the $52 \mathrm{Ab} \epsilon$ antibody (1:4000; Sigma) or peroxidaseconjugated anti-mouse IgG for mAb 88B (1:4000; Sigma) for $2 \mathrm{hr}$ at room temperature, washed, incubated in chemiluminescence solution (NEN, Boston, MA), and exposed to Kodak x-ray film (Eastman Kodak, Rochester NY). For the $\epsilon$ subunit degradation, samples from days 1, 2, 4, 7, and 10 after ${ }^{125}$ I-BTX labeling as well as the serial dilution of pooled AChR extract at 1:1, 1:2, 1:4, and 1:8 were run. The PVDF membrane was incubated in blocking buffer $(5 \mathrm{mg} / \mathrm{ml} \mathrm{BSA}$ and $1 \%$ SDS or $4 \%$ nonfat milk and $0.1 \%$ Tween 20 in Tris buffer, $\mathrm{pH} \mathrm{7.2)} \mathrm{for} 15 \mathrm{~min}-2 \mathrm{hr}$ at room temperature. The membrane was then incubated for $12 \mathrm{hr}$ at $4^{\circ} \mathrm{C}$ with the anti- $\epsilon$ subunit antibody $52 \mathrm{Ab} \epsilon(1: 1000-1: 2000)$. The primary antibody was removed, and the membrane was washed three times for 5 min each with $50 \mathrm{ml}$ of blocking buffer and then incubated for $2 \mathrm{hr}$ at room temperature with ${ }^{125} \mathrm{I}$ anti-rabbit $\mathrm{IgG}$ (1:200; Amersham) in $10 \mathrm{ml}$ of blocking buffer. The secondary antibody was removed, and the membrane was rinsed in $50 \mathrm{ml}$ of blocking buffer and washed three times in $100 \mathrm{ml}$ of Tris buffer plus 1\% SDS before exposure for 1-3 d to BioMax MS film (Kodak) at $-70^{\circ} \mathrm{C}$. The autoradiography film was developed, and video-based densitometry was performed. Degradation of the $\epsilon$ subunit was determined by plotting the residual densitometric values from Western blots of each time point calibrated by the standard curve obtained from the serial dilutions. The values were then normalized to the day 1 value and plotted on a semilogarithmic graph. Half-life values were obtained from the slopes of the exponential fits to the data.

\section{Cell transfection}

HEK 293 cells (CRL 1573; American Type Culture Collection, Rockville, MD) were transfected with different AChR subunits. The subunits were cloned into an expression plasmid containing a cytomegalovirus promoter (pBEX1; British Biotechnologies Ltd., Oxford, UK). The mouse AChR subunits $\alpha, \beta, \delta$, and $\epsilon$ were a gift from Dr. Steven Heinemann (Salk Institute, La Jolla, CA). Cells were plated in $35 \mathrm{~mm}$ cell culture dishes in DMEM, supplemented with $10 \%$ fetal calf serum,

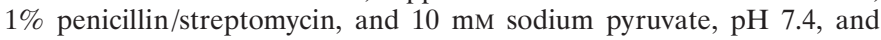
incubated in $90 \%$ air $/ 10 \% \mathrm{CO}_{2}$ at $37^{\circ} \mathrm{C}$. Subconfluent cells were transfected by a standard calcium phosphate precipitation method (Wigler et al., 1979) using $1.25 \mu \mathrm{g}$ of total DNA (with different subunit combinations) and $0.12 \mu \mathrm{g}$ of pRSVT (a plasmid expressing the Simian virus 40 large $\mathrm{T}$ antigen driven) (Lebkowski et al., 1985). Transfected cells were incubated for $48 \mathrm{hr}$, washed, and harvested in $100 \mathrm{~mm}$ phosphate buffer, $\mathrm{pH} 7.4$, with $0.5 \mathrm{~mm}$ EDTA and spun at $2000 \times \mathrm{g}$ for $10 \mathrm{~min}$. To lyse the cells, the precipitate was resuspended in $5 \mathrm{ml}$ of double-distilled $\mathrm{H}_{2} \mathrm{O}$ with $0.5 \mathrm{~mm}$ PMSF and frozen at $-80^{\circ} \mathrm{C}$ for $1 \mathrm{hr}$. The cells were then thawed to room temperature and centrifuged at $15,000 \times g$ for at least 1 $\mathrm{hr}$ to collect the membrane fragments, which were dissolved in SDS sample buffer, boiled for $10 \mathrm{~min}$, and assayed by Western analysis.

\section{Antibodies}

Anti- $\alpha$ mAb 155 and anti- $\epsilon$ mAb 168 were gifts from Dr. S. Tzartos (Pasteur Institute, Athens, Greece); the anti- $\gamma / \delta \mathrm{mAb} 88 \mathrm{~B}$ was a gift from Dr. S. C. Froehner (University of North Carolina, Chapel Hill, NC); $\mathrm{mAb} 88 \mathrm{~B}$ was also purchased from Affinity Bio Reagent Inc. (Golden, $\mathrm{CO}$ ). Because the anti- $\epsilon$ mAb 168 did not give a sufficient signal for the Western analysis, a new anti- $\epsilon$ subunit antibody $(52 \mathrm{Ab} \epsilon)$ was generated at Research Genetics (Huntsville, AL) in rabbits injected with a synthesized 15 amino acid peptide (ARR ASSVGILLRAEE) from the intracellular loop of the rat $\epsilon$ subunit (amino acids 369-383).

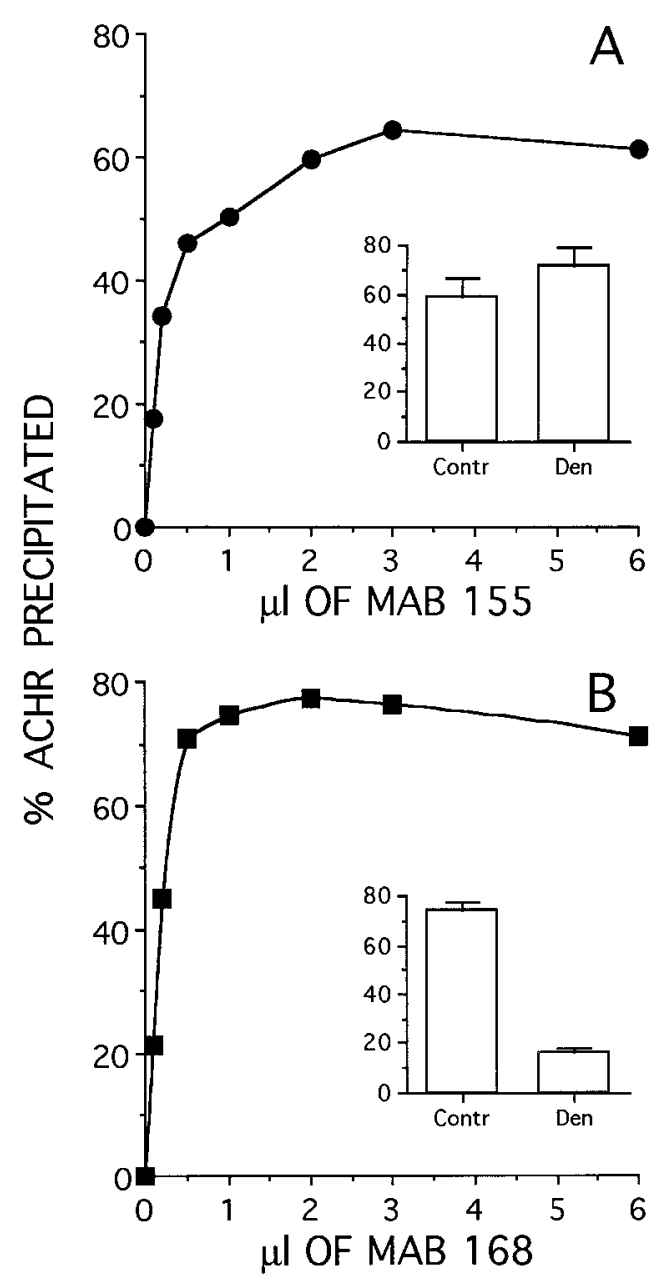

Figure 1. Efficiency of immunoprecipitation of adult AChR by the anti- $\alpha$ subunit mAb $155(A)$ or the anti- $\epsilon$ subunit mAb $168(B)$. Innervated soleus muscles were labeled in vitro with ${ }^{125}$ I-BTX, extracted with $2 \%$ Triton X-100, and immunoprecipitated with mAbs 155 and 168 (see Materials and Methods). The amount of immunoprecipitated radioactivity (AChR) was expressed as the percentage of total activity in the reaction mixture. The insets compare the maximum percent immunoprecipitated from innervated control (Contr) and $7 \mathrm{~d}$ denervated (Den) soleus muscle at saturating concentrations of antibodies.

\section{RESULTS \\ Antibody specificity}

The subunit specificity of the anti- $\epsilon$ subunit mAb 168 and anti- $\alpha$ subunit mAb 155 used in this study has already been established (Tzartos et al., 1986; Nelson et al., 1992; Engel et al., 1993; Green and Claudio, 1993). Conditions for maximum immunoprecipitation by the subunit-specific mAbs were established using AChR extracted from adult soleus muscles prelabeled in vitro with ${ }^{125} \mathrm{I}$ BTX and immunoprecipitated with increasing concentrations of either of the two mAbs. Saturating amounts of mAb 155 and mAb 168 immunoprecipitated, respectively, $59 \pm 8$ and $74 \pm 4 \%$ of the labeled receptors (Fig. 1 $A, B$ ). This immunoprecipitation efficiency was maintained when the muscle extracts were diluted up to 100 -fold. The less than complete precipitation attained with mAb 168 was not attributable to the presence in the extract of different subsets of $\epsilon$-AChR, because the same efficiency was obtained when the supernatant remaining after immunoprecipitation was again incubated with the same amount of antibody. Similar results were obtained with the anti- $\epsilon$ subunit mAb 154 
(Tsartos et al., 1986) and when the diaphragm (instead of the soleus) muscle was used (data not shown). In denervated soleus muscles, mAb 168 immunoprecipitated only $16 \pm 2 \%$ of labeled receptors because of the large increase in extrajunctional $\gamma$ AChR (Fig. 1B, inset). These mAbs were then used for the immunoprecipitation studies to determine $\epsilon$-AChR-specific degradation rates (to be described below).

To test the specificity of the new anti- $\epsilon$ subunit antibody, $52 \mathrm{Ab} \epsilon$, we transfected HEK 293 cells with cDNAs of AChR $\alpha, \beta$, $\epsilon$, and $\delta$ subunits singly or in various combinations. Although the antibody was raised against a 15 amino acid peptide from the $\epsilon$ subunit, this sequence shows a 53\% homology (8 of 15 amino acids) with the $\delta$ subunit. To test the possibility of cross-reactivity, HEK 293 cells were transfected with cDNAs coding for the $\alpha$ subunit and either the $\epsilon$ or the $\delta$ subunit. In this case, $\alpha \delta$ or $\alpha \epsilon$ dimers, the intermediates of AChR assembly (Blount and Merlie, 1991), were expected to form. The membrane preparations of the cells were separated on an SDS gel, transferred to a PVDF membrane, and probed by the anti- $\epsilon$ 52Ab $\epsilon$ antibody, the anti- $\gamma / \delta$ $88 \mathrm{~B} \mathrm{mAb}$, or the preimmune serum or $\epsilon$ peptide-absorbed $52 \mathrm{Ab} \epsilon$.

Figure 2, $A$ and $B$, shows the Western blot results. The $52 \mathrm{Ab} \epsilon$ antibody specifically recognized a major band (of $\sim 60 \mathrm{kDa}$ ) from cells transfected with the $\epsilon$ plus the other three AChR subunit cDNAs as well as with $\alpha \epsilon$ but did not recognize anything from the cells transfected with the $\alpha \delta$. By contrast, the anti- $\gamma / \delta$ subunit $\mathrm{mAb} 88 \mathrm{~B}$ recognized proteins only from the cells that were transfected with either all four subunits of AChRs or only $\alpha \delta$ but failed to recognize anything from cells transfected with $\alpha \epsilon$ or the untransfected controls. These results showed that the $52 \mathrm{Ab} \epsilon$ polyclonal antibody specifically and selectively recognized the $\epsilon$ subunit of rat AChRs.

\section{Estimates of total AChR degradation by ${ }^{125}$ I-BTX release into medium}

In myotubes labeled with ${ }^{125} \mathrm{I}-\mathrm{BTX}$, the AChR degradation rate was measured by means of the release of radioactivity in the culture medium as described previously (O'Malley et al., 1993). The data are shown as the decrease in cell-associated specific radioactivity, normalized to the value at the time of labeling (Figs. 3A, 4D). Regardless of whether the cells were labeled with ${ }^{125}$ I-BTX on day 4 or up to day 13 after plating, the decay of cell-associated radioactivity was biphasic, thus indicating the presence of two AChR populations with different degradation rates. The rapid component $(\mathrm{Rr})$ had a half-life of $\sim 1 \mathrm{~d}$ and constituted $\sim 90 \%$ of the total receptor. The remaining AChR population had a half-life of $\sim 3-4 \mathrm{~d}$. When cells were labeled with ${ }^{125}$ I-BTX $13 \mathrm{~d}$ after plating, the slow component (Rs) had a more variable halflife, occasionally reaching $8.1 \mathrm{~d}$. This occasional greater stabilization of half-life in older cultures is consistent with results reported previously (O’Malley et al., 1993; Salpeter et al., 1993).

\section{Estimates of total and $\epsilon$-AChR by immunoprecipitation}

${ }^{125}$ I-BTX labeled AChRs were extracted from myotubes as described in Materials and Methods and were immunoprecipitated with saturating amounts of either anti- $\alpha$ subunit mAb 155 or anti- $\epsilon$ subunit mAb 168. The relative amount of total radioactivity immunoprecipitated at each time point after ${ }^{125}$ I-BTX labeling was expressed as a percentage of the relative value obtained with each antibody on the day of labeling (degradation day 0 ).

The results obtained from AChRs labeled $8 \mathrm{~d}$ after plating and immunoprecipitated with either the anti- $\alpha$ or anti- $\epsilon$ subunit antibodies are shown in Figure $3, B$ and $C$, and indicate that the
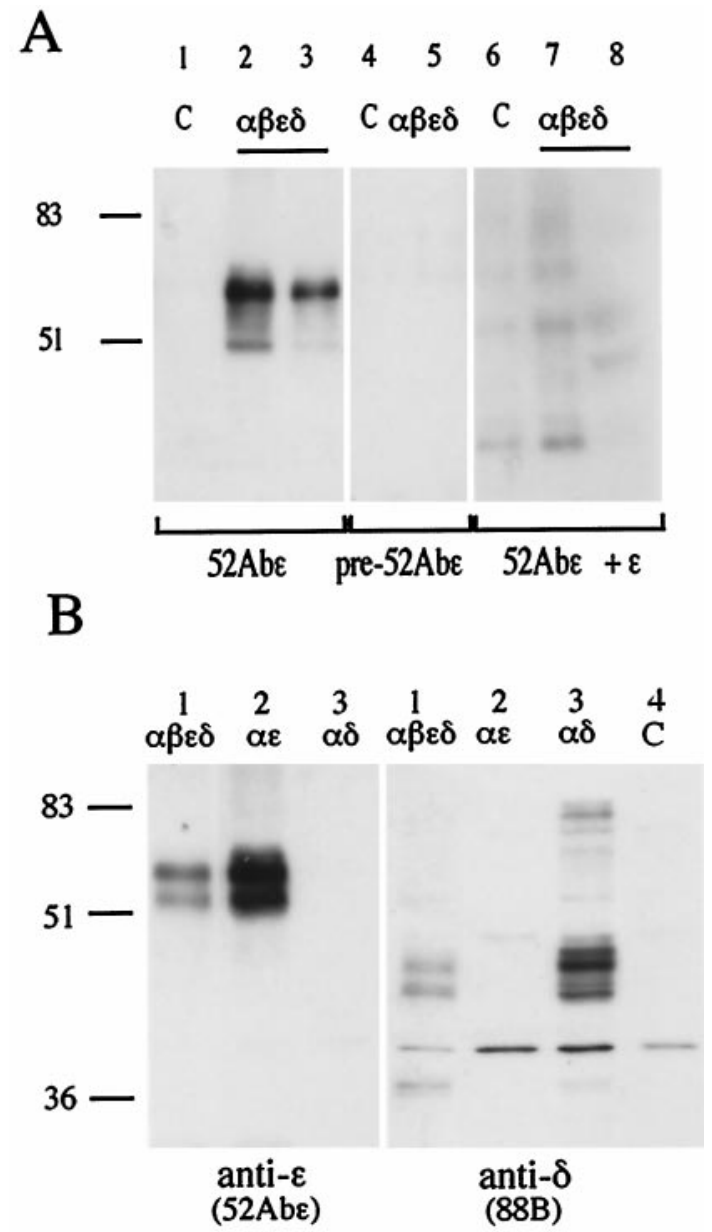

Figure 2. The anti- $\epsilon 52 \mathrm{Ab} \epsilon$ polyclonal antibody specifically recognizes the $\epsilon$ AChR subunit on Western blots. A, HEK 293 kidney fibroblast cells were transfected with cDNAs coding for the $\alpha \beta \epsilon \delta$ subunits $(2: 1: 1: 1)$ of rat AChR (lanes 2, 3, 5, 7, 8) or left as untreated controls (lanes 1, 4, 6). The $52 \mathrm{Ab} \epsilon$ antibody recognized a major band at molecular weight $\sim 60 \mathrm{kDa}$ in the $\epsilon$-containing AChR-transfected cells (lanes 2, 3) but not in the control nontransfected cells (lane 1). No bands were seen with preimmune serum (lanes 4,5 ) or after preabsorption of the 52Ab $\epsilon$ antibody with the $\epsilon$ peptide (lanes 6-8). Numbers on the left correspond to molecular mass standards (in kilodaltons). B, Samples obtained from HEK 293 cells transfected with $\alpha \beta \epsilon \delta$ (2:1:1:1), $\alpha \epsilon$ (2:1), or $\alpha \delta$ (2:1) cDNAs were incubated with either the anti- $\epsilon$ subunit $52 \mathrm{Ab} \epsilon$ antibody or the anti- $\gamma / \delta$ subunit $\mathrm{mAb} 88 \mathrm{~B}$. The $52 \mathrm{Ab} \epsilon$ antibody recognized a major bands of $60 \mathrm{kDa}$ in the cells transfected with $\alpha \beta \epsilon \delta$ and $\alpha \epsilon$ but none in the cells transfected with $\alpha \delta$. mAb 88B specifically immunodecorated the lanes loaded with membrane preparations obtained from the cells transfected with $\alpha \beta \epsilon \delta$ and $\alpha \delta$ but not from cells transfected with $\alpha \epsilon$ or the nontransfected control. $(C)$.

degradation of the receptors recognized by the two subunitspecific mAbs followed different kinetics. The decrease in radioactivity immunoprecipitated by the anti- $\alpha$ subunit mAb 155, representing degradation of the total $\mathrm{AChR}$ population, was biphasic (Fig. $3 B$ ) and very similar to the degradation rates obtained by the method of radioactive release described above (Figs. 3A, 4D). The fast component was $\sim 90 \%$ and had a half-life of $\sim 0.8 \mathrm{~d}$. The slow component comprised $\sim 10 \%$ of the receptors and had a half-life of $2.6 \pm 0.6 \mathrm{~d}$. Similar results were obtained from the cells labeled $13 \mathrm{~d}$ after plating. In these cells, the half-life of the slow component was $3.1 \pm 0.3 \mathrm{~d}$ when measured by immunoprecipitation, compared with $3.9 \pm 0.9 \mathrm{~d}$, as in Figure $4 D$, when determined by toxin degradation on parallel dishes (data not 

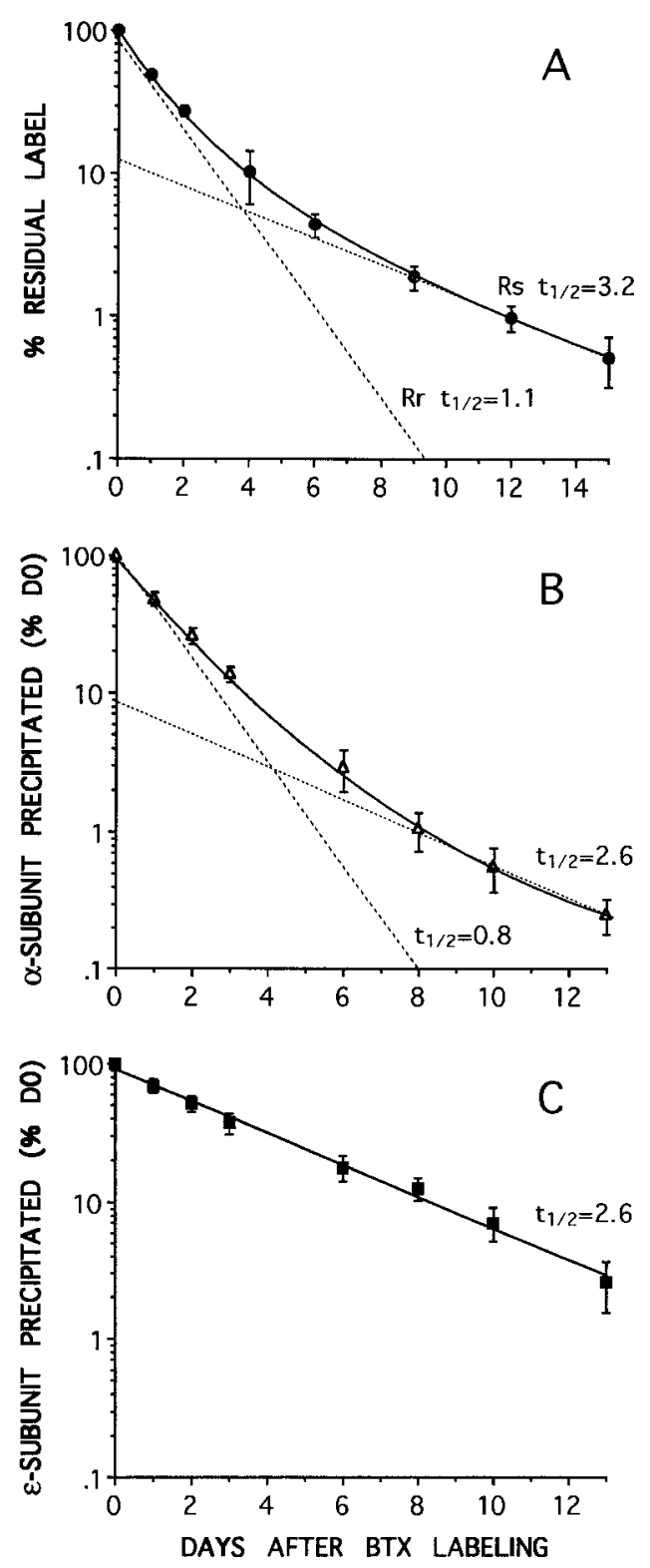

Figure 3. AChR degradation rates measured by either ${ }^{125}$ I-BTX release $(A)$ or by subunit-specific immunoprecipitation with anti- $\alpha(B)$ or anti- $\epsilon$ (C) subunit mAbs. At each of the time points data are expressed as a percentage of the radioactivity immunoprecipitated from the cells at the time of labeling with ${ }^{125}$ I-BTX (day 0). Biphasic degradation curves for total AChRs are obtained both by ${ }^{125}$ I-BTX release $(A)$ or by anti- $\alpha$ subunit mAb 155 immunoprecipitation $(B)$. In each case the best fit to the data (solid curve) consists of the sum of two components (dashed lines): a slow component (Rs), with a $t_{1 / 2}$ value of $\sim 3 \mathrm{~d}(3.2$ and $2.6 \mathrm{~d}$ ), and a fast component $(\mathrm{Rr})$, with a $t^{1 / 2}$ of $\sim 1 \mathrm{~d}(1.1$ and $0.8 \mathrm{~d})$. The receptor immunoprecipitated by the anti- $\epsilon$ subunit mAb $168(C)$ degrades as a single exponential (solid line) with a $t_{1 / 2}$ of $2.6 \mathrm{~d}$, similar to the slow components in $A$ and $B$. No fast component is seen. The values are the means $\pm \mathrm{SD}$ of at least three different experiments. The data in $B$ and $C$ were obtained from the same sets of cells.

shown). Unlike the decrease in radioactivity immunoprecipitated with the anti- $\alpha$ subunit mAb 155, the decrease in radioactivity immunoprecipitated by the anti- $\epsilon$ subunit mAb 168 followed a first-order decay (Fig. $3 C$ ), indicating the presence of only one population. The half-life values obtained with mAb 168 were very similar to those of the slow component estimated with mAb 155 in the same sets of cells (Fig. 3, compare $B, C$ ). No fast component was seen. This was true regardless of the age of the cell at the time of labeling with radioactive BTX (data not shown).

\section{$\epsilon-A C h R$ degradation estimated by Western blot analysis}

When AChRs, extracted from cultured muscle cells at various time after labeling with ${ }^{125} \mathrm{I}$-BTX in culture, were probed on Western blots with the anti- $\epsilon$-subunit polyclonal antibody 52Ab $\epsilon$, similar degradation results were obtained as after immunoprecipitation by the anti- $\epsilon$ subunit mAb 168 . The $\sim 60 \mathrm{kDa}$ band recognized by the polyclonal antibody $52 \mathrm{Ab} \epsilon$ decreased with intensity over time after degradation day 0 (Fig. 4A). Comparison with band densities from a serial dilution of the same samples (Fig. 4B) confirmed that the assay was linear and thus quantitative. Densitometric values were plotted on a semilog plot and fitted by either a single or double exponential. The best fit was given by a single exponential with a half-life of $3.0 \mathrm{~d}$ (Fig. $4 C$ ), similar to the 2.6 day half-life of the immunoprecipitates obtained
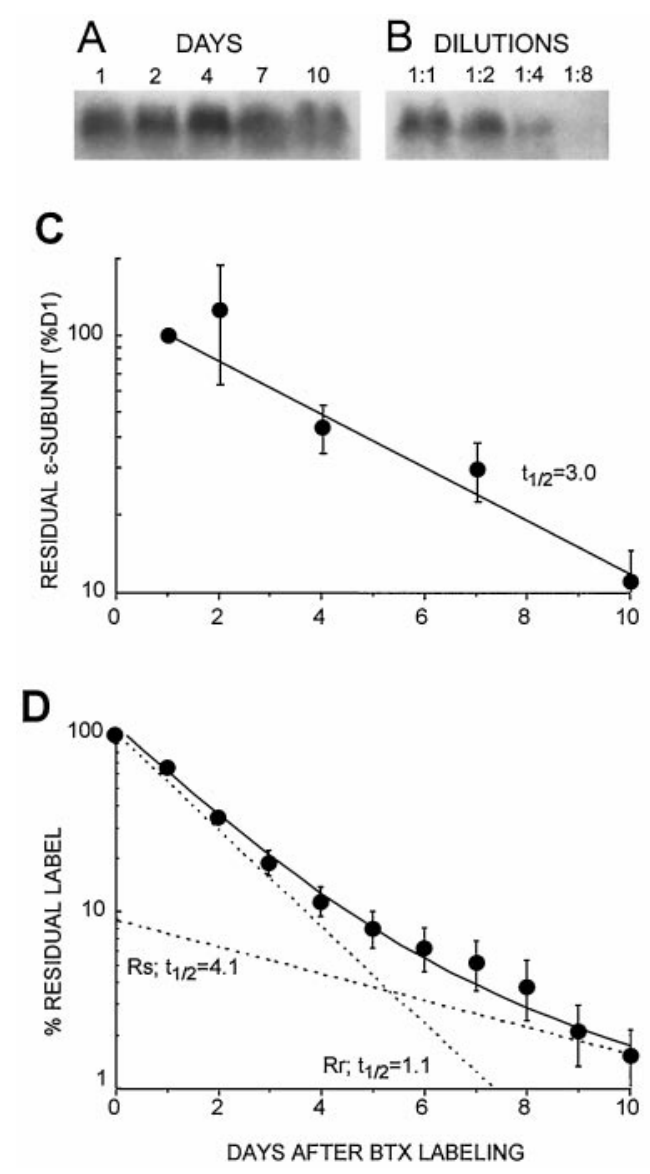

Figure 4. Western blot analysis of $\epsilon$-AChR degradation in cultured muscle cells compared with total AChR degradation measured by ${ }^{125} \mathrm{I}-$ BTX release. $A$, Sample of Western blots at different times after BTX labeling, showing the $\sim 60 \mathrm{kDa}$ immunogenic band recognized by the anti- $\epsilon$ subunit antibody $52 \mathrm{Ab} \epsilon$ (as in Fig. 2). B, Successive dilutions of antigen established linearity of the Western blot response. $C$, A plot of the density of the anti- $\epsilon$ subunit bands, decreasing with time after labeling, gives a single exponential decay with a half-life of $\sim 3.0 \mathrm{~d}(n=3$ experiments), similar to the slow components in Figure 3, $A$ and $B$. No fast component is seen. $D$, Residual label from parallel plates assayed by ${ }^{125}$ I-BTX release gives a double-exponential fit for total AChR (as in Fig. $3 A, B)$, revealing two AChR populations with slow, Rs, and fast, $\mathrm{Rr}$, components having $t_{1 / 2}$ values of 4.1 and $1.1 \mathrm{~d}$, respectively. 
with the anti- $\epsilon$ subunit mAb 168 (Fig. 3C) and to the slow component from the anti- $\alpha$ subunit precipitate (Fig. $3 B$ ). In all cases the material recognized by the anti- $\epsilon$ subunit antibodies resembles the Rs obtained from ${ }^{125}$ I-BTX release degradation curves (Figs. $3 A, 4 D$ ) and in previous studies. Furthermore, no evidence for the faster $1 \mathrm{~d}$ half-life, typical of $\mathrm{Rr}$ AChRs, was seen with anti- $\epsilon$ subunit degradation curves.

It is possible that unassembled $\epsilon$ subunit would not be removed from the AChR extracted by the BTX beads (see Materials and Methods) and act as a contaminant in the epsilon degradation estimations. However, if there was indeed a contaminating level of unlabeled epsilon subunit that was not removed with the BTX beads, this would be seen in the Western analysis as a persistent signal that does not decay and would be seen at long time points as a line parallel to the $x$-axis. Yet such a persistent signal was not seen. Indeed the loss of epsilon subunit is best described with a single exponential decay. Therefore, the use of BTX beads to remove unlabeled AChRs does not result in significant contamination by unassembled epsilon subunit.

\section{Stabilization of Rs and $\epsilon$-AChRs by dibutyryl-cAMP}

As a further indication that $\epsilon$-AChR molecules are exclusively included in the Rs pool, degradation was measured by ${ }^{125}$ I-BTX release and by $\mathrm{mAb} 168$ immunoprecipitation in cells treated with $1 \mathrm{mM}$ dibutyryl-cAMP (dB-cAMP), a condition that increases Rs half-life. As reported previously (Shyng et al., 1991; O’Malley et al., 1993), this treatment caused the half-life of the slow component to increase $\sim 1$.7-fold both by ${ }^{125}$ I-BTX loss and by anti- $\epsilon$ immunoprecipitation (Fig. 5A,B). No change occurred in the degradation of the fast component that retained its $1 \mathrm{~d}$ half-life.

\section{DISCUSSION}

The present study aims at understanding whether the substitution of the $\epsilon$ for the $\gamma$ subunit in the muscle AChR is involved in regulating AChR degradation behavior. To date it has been unequivocably established that this subunit substitution affects the physiological characteristics of the receptor, yet all attempts to assign degradative characteristics to this subunit switch have resulted in conflicting conclusions.

The most serious argument against the hypothesis that subunit composition affects AChR degradation had for some time come from older studies showing that AChRs are more stable than embryonic receptors already at birth (Steinbach et al., 1979; Reiness and Weinberg, 1981; Cohen et al., 1990; for review, see Hall and Sanes, 1993), whereas the expression of the $\epsilon$ subunit starts days later (Sakmann and Brenner, 1978; Vicini and Schuetze, 1985; Gu and Hall, 1988; Gundersen et al., 1993; Missias et al., 1996). Furthermore, there is no precise temporal coincidence between changes in channel properties (a clear indication of subunit switch) and turnover rates during the maturation of ectopic NMJs (Reiness and Weinberg, 1981; Brenner and Sakmann, 1983) and of muscle in culture (Brehm et al., 1983).

Later studies attempting to test this hypothesis directly, using heterologous nonmuscle expression systems, also have produced conflicting results. Some studies have found that the degradation rates for $\epsilon$-AChRs were slower than for $\gamma$-AChRs (Gu et al., 1990; Jayawickreme and Claudio, 1994). Other studies found no differences (Kopta and Steinbach, 1994; Liu et al., 1994). However, the absolute half-lives of the expressed AChRs were significantly different in these studies than seen in muscle. The cellular "environment" of the muscle thus may also play a role in defining the metabolic properties of the AChRs.
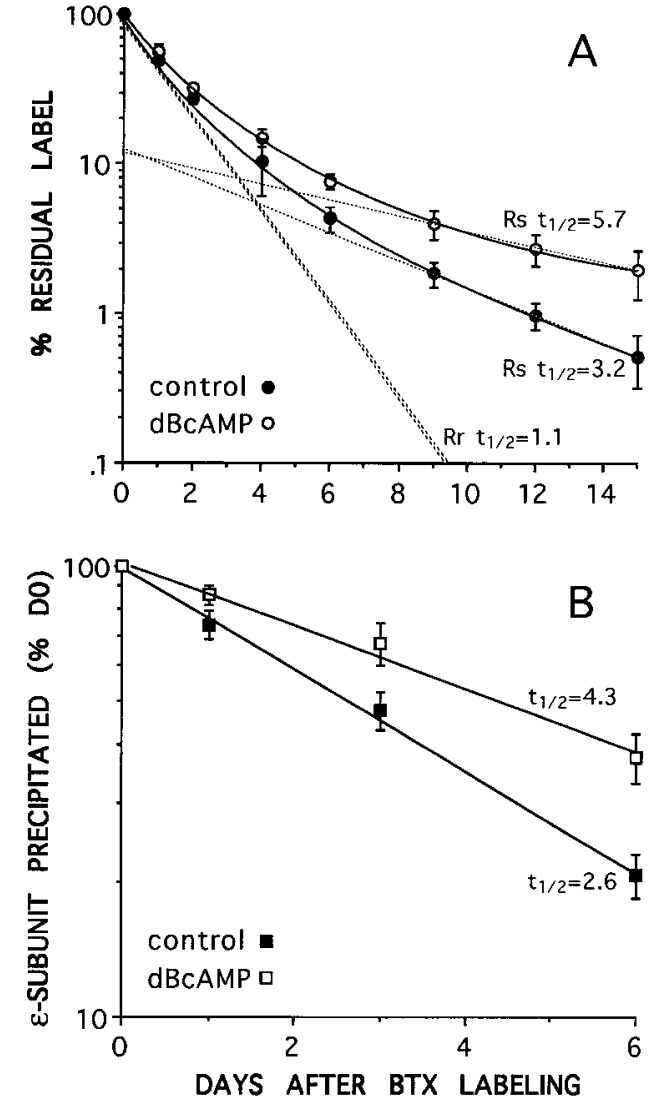

Figure 5. Dibutyryl-cAMP $(d B c A M P)$ slows the degradation rate of the slow component, Rs, when measured by ${ }^{125}$ I-BTX release $(A)$ and of the material immunoprecipitated by the anti- $\epsilon$ subunit mAb $168(B)$. Incubation in dB-cAMP increases the $t^{1 / 2}$ of Rs (from 3.2 to $5.8 \mathrm{~d}$ ) and that of the $\epsilon$-subunit (from 2.7 to $4.3 \mathrm{~d}$ ) but does not affect the $t^{1 / 2}$ of $\mathrm{Rr}$, which remains at $1.1 \mathrm{~d}(A)$. Dashed lines in $A$ give the two component exponential decays for $\mathrm{Rs}$ and $\mathrm{Rr}$ (see Materials and Methods), which when summed give the best fit to the experimental data (solid lines) for each condition. The two exponentials for $\mathrm{Rr}$ overlap for control and $\mathrm{dB}-$ cAMP-treated cells.

To distinguish AChR species on the basis of their degradation properties, the terms Rs (slowly degrading) and $\mathrm{Rr}$ (rapidly degrading) were introduced (Shyng and Salpeter, 1990). Subsequently, as more information regarding the different behavior patterns and responses to external factors became available, the terms have acquired more selective meaning. Especially is it important to recognize that degradation rate alone is an insufficient criterion for judging which isoform a receptor belongs to. Basically, the Rs is the adult AChR expressed at innervated NMJs, at which it is very stable with a slow degradation rate $\left(t_{1} / 2\right.$ $=\sim 10 \mathrm{~d})$. At the other extreme is the Rr AChR, synthesized in embryonic, cultured, or denervated adult muscle, which usually has a rapid degradation rate $\left(t_{1 / 2}=\sim 1 \mathrm{~d}\right)$. Although the exact degradation half-lives differ somewhat for different muscles and animals, this clear distinction between the slowly degrading innervated adult, Rs, AChR and the predominantly rapidly degrading embryonic, $\mathrm{Rr}$, AChR is characteristic of all mammalian muscles studied to date. Based on all the available evidence, we conclude that Rs and $\mathrm{Rr}$ do not interconvert but are independently synthesized molecules that replace each other during development, denervation, and reinnervation (Shyng and Salpeter 1989, 1990; Stiles and Salpeter, 1997). 
In addition to the two distinct and extreme half-lives of 10 and $1 \mathrm{~d}$, intermediate and overlapping degradation rates have been reported. These (for review, see Salpeter and Loring, 1985; Hall and Sanes, 1993) are seen after denervation, during development and reinnervation (M. M. Salpeter and M. Szabo, unpublished observations). In principle these could be accelerated Rs, stabilized $\mathrm{Rr}$, a mixture of the two, or even distinct populations. In each case their nature must be established independently. Often it is obvious what these intermediate AChRs are. For instance, when Rs AChRs were studied by labeling the AChRs of innervated NMJs, these labeled Rs AChRs were seen to acquire an intermediate half-life after denervation (with a $t_{1 / 2}$ of $\sim 3-4 \mathrm{~d}$ ), becoming accelerated Rs, and were restabilized on reinnervation (Levitt and Salpeter, 1981; Salpeter et al., 1986; Andreose et al., 1993). On the other hand, the metabolic properties of $\mathrm{Rr}$ synthesized at the denervated NMJ were studied by radioactive labeling after saturating the AChRs, preexisting before denervation, with nonradioactive toxin (Shyng and Salpeter, 1990).

Because Rs and Rr can be labeled independently, various criteria in addition to degradation rate were then applied to distinguish between these two species. One method was to analyze their responses to stabilizing factors. It was found that Rs and $\mathrm{Rr}$ respond differently to electrical stimulation and thus muscle activity. Electrical stimulation keeps Rs stable by preventing its postdenervation acceleration and downregulates the expression of Rr without altering its half-life (Fumagalli et al., 1990; Andreose et al., 1993). Accelerated Rs but not Rr can be stabilized by cAMP (Shyng et al., 1991; O’Malley et al., 1993). On the other hand, $\mathrm{Rr}$ can be stabilized by activation of $\mathrm{P}_{2}$ purinergic receptors (O'Malley et al., 1997), whereas cAMP as well as high cytosolic calcium concentration counteract this stabilizing effect on Rr (O’Malley et al., 1997). Degradation rate and sensitivity to stabilizing factors can therefore be combined to identify a metabolic population of AChR even when both are present simultaneously and cannot be distinguished by differential labeling.

The present study provides the first direct measure of the degradation rate and response to $\mathrm{dB}-\mathrm{CAMP}$ of an $\mathrm{AChR}$ population characterized by its subunit composition. We have focused on the $\epsilon$ subunit-containing AChR for which immunological tools were available. Unfortunately we have not been able to generate, nor have we found elsewhere, any appropriate anti- $\gamma$ subunit antibodies to perform similar studies for the $\gamma$-AChRs. This study was performed in aneurally cultured rat myotubes, because they contain clearly distinguishable $\mathrm{Rr}$ and $\mathrm{Rs}$ with classical sensitivity to cAMP, and, unlike heterologous expression systems, they provide the natural cellular environment for muscle receptors. They closely resemble long-term denervated NMJs containing (in a ratio of 9:1) both $\mathrm{Rr}$ and accelerated Rs (Shyng and Salpeter, 1990; O'Malley et al., 1993) as well as $\epsilon$ - and $\gamma$-AChRs (Siegelbaum et al., 1984; Brenner et al., 1990; Goldman et al., 1991; Pinset et al., 1991; Shepherd and Brehm, 1994). The main observations of our study are (1) when the anti- $\epsilon$ subunit-specific antibodies mAb 168 and $52 \mathrm{Ab} \epsilon$ were used to measure the degradation rate of $\epsilon$-AChRs labeled with ${ }^{125} \mathrm{I}$-BTX, the loss of antigen with time was fit by a single exponential with a $t_{1 / 2}$ of $\sim 3 \mathrm{~d}$, similar to the $t_{1 / 2}$ of the accelerated Rs in the same cells; and (2) treatment with dB-cAMP increased both $\epsilon$-AChR and Rs half-lives by a similar factor, whereas the treatment did not affect $\mathrm{Rr}$ degradation.

For unknown reasons, the degradation half-lives obtained with the antibodies were slightly shorter than those obtained by ${ }^{125}$ I-BTX release, yet their basic characteristics were the same. Taken together, our results unequivocally show that, in cultured rat myotubes, $\epsilon$-AChR molecules are included in the Rs but not the $\mathrm{Rr}$ pool. Selection of $\epsilon$-AChR to the Rs pool with its ability to be stabilized by cAMP mediated by protein kinase A (PKA) (Xu and Salpeter, 1995) may be attributable to the presence of critical residues exposed in the $\epsilon$ subunit, possibly related to the PKA phosphorylation site, which exists on the $\epsilon$ but not the $\gamma$ subunits of mammalian AChRs (Miles et al., 1989). Interaction with cytoskeletal proteins may also be an important factor. For instance, in the mutant $m d x$ mouse lacking dystrophin, the adult innervated Rs AChRs are permanently in an accelerated state (Xu and Salpeter, 1997).

Our data do not exclude the possibility that $\gamma$-AChR may also be included among the slowly degrading population under certain conditions. On the other hand, the fact that all the $\epsilon$-AChRs are in the Rs pool indicates that subunit composition influences the mechanism whereby receptor molecules are sorted to their metabolic pools.

A fascinating picture is now emerging based on the data provided by this study and the recent observation that the rapidly degrading $\operatorname{Rr}$ (presumably $\gamma$-AChRs) on cultured muscle cells can be stabilized by ATP, an effect that is reversed or modified by high cytosolic calcium concentration or cAMP (O'Malley et al., 1997). Thus, in the presence of the nerve that releases ATP together with transmitter (Silinski, 1975), a population of stabilized $\gamma$-AChRs could be created in the early postnatal muscle. The increased metabolic stability participates in increasing the postsynaptic receptor number and thus in strengthening neuromuscular transmission. As receptor density increases during NMJ maturation, so will $\mathrm{Ca}^{2+}$ influx (Leonard and Salpeter, 1979, 1982; Vernino et al., 1994). Increased intracellular $\mathrm{Ca}^{2+}$ should lead to destabilization of the ATP-stabilized $\gamma$-AChRs (O'Malley et al., 1997) as well as downregulation of these embryonic receptors (Rubin, 1985; Duclert and Changeaux, 1995) (J. O'Malley and M. M. Salpeter, unpublished observations), which are then rapidly and efficiently replaced by the adult $\epsilon$-AChR. In this case, the replacement of the $\gamma$ by the $\epsilon$ subunit would contribute not only to the changes in the electrical properties of the synapse but also to its stability. It is tempting to speculate that the developmental changes in subunit expression seen for various neurotransmitter receptors in the CNS have a similar significance.

\section{REFERENCES}

Andreose J, Xu R, Lømo T, Salpeter MM (1993) Degradation of two AChR populations at rat neuromuscular junction: regulation by electrical stimulation. J Neurosci 13:3433-3438.

Blount P, Merlie JP (1991) Characterization of an adult muscle acetylcholine receptor subunit by expression in fibroblasts. J Biol Chem 266:14692-14696.

Brehm P, Yeh E, Patrick J, Kidokoro Y (1983) Metabolism of acetylcholine receptors on embryonic amphibian muscle. J Neurosci 3:101-107.

Brenner HR, Sakmann B (1983) Neurotrophic control of channel properties at neuromuscular synapses of rat muscle. J Physiol (Lond) 337:159-171.

Brenner HR, Witzemann V, Sakmann B (1990) Imprinting of acetylcholine receptor messenger RNA accumulation in mammalian neuromuscular synapses. Nature 344:544-547.

Cohen MW, Frair PF, Cantin C, Herbert G (1990) Developmental changes in the half life of acetylcholine receptors in the myotomal muscle of Xenopus laevis. J Physiol (Lond) 426:281-296.

Duclert A, Changeaux J-P (1995) Acetylcholine receptor gene expression at the developing neuromuscular junction. Physiol Rev 75:339-368.

Engel AG, Hutchinson DO, Nakano S, Murphy L, Griggs RC, Gu Y, Hall ZH, Lindstrom J (1993) Myasthenic syndromes attributed to mutation affecting the epsilon subunit of the acetylcholine receptor. In: Myasthenia gravis and related disorders: experimental and clinical aspects (Penn AS, Richman DP, Ruff RL, Lennon VA, eds), pp 496-508. New York: New York Academy of Sciences. 
Froehner SC, Douville K, Klink S, Culp WJ (1983) Monoclonal antibodies to cytoplasmic domains of acetylcholine receptor. J Biol Chem 258:7112-7120.

Fumagalli G, Balbi S, Cangiano A, Lømo T (1990) Regulation of turnover and number of acetylcholine receptors at neuromuscular junction. Neuron 4:563-569.

Goldman D, Brenner HR, Heinemann S (1988) Acetylcholine receptor $\alpha, \beta, \gamma, \delta$ subunit mRNA levels are regulated by muscle activity. Neuron 3:219-228.

Goldman D, Carlson BM, Staple J (1991) Induction of adult-type nicotinic acetylcholine receptor gene expression in non innervated regenerating muscle. Neuron 7:649-658.

Gotti C, Conti-Tronconi BM, Raftery M (1982) Mammalian muscle acetylcholine receptor purification and characterization. Biochemistry 21:3148-3154.

Green WN, Claudio T (1993) Acetylcholine receptor assembly: subunit folding and oligomerization occur sequentially. Cell 74:57-69.

Gu Y, Hall ZW (1988) Immunological evidence for a change in subunits of the acetylcholine receptor in developing and denervated rat muscle. Neuron 1:117-125.

Gu Y, Franco AJ, Gardner PD, Lansman JB, Forsayeth JR, Hall ZW (1990) Properties of embryonic and adult muscle acetylcholine receptors transiently expressed in COS cells. Neuron 5:147-157.

Gundersen K, Sanes JR, Merlie JP (1993) Neural regulation of muscle acetylcholine receptor $\epsilon$ - and $\alpha$-subunit gene promoters in transgenic mice. J Cell Biol 123:1535-1544.

Hall ZW, Sanes JR (1993) Synaptic structure and development: the neuromuscular junction. Cell/Neuron [Suppl] 72:10:99-121.

Jayawickreme SP, Claudio T (1994) Forskolin stabilizes epsilon subunitcontaining acetylcholine receptors. Brain Res Mol Brain Res 26:293-298.

Karlin A (1993) Structure of the nicotinic acetylcholine receptors. Curr Opin Neurobiol 3:299-309.

Kopta C, Steinbach JH (1994) Comparison of mammalian adult and fetal nicotinic acetylcholine receptors stably expressed in fibroblasts. J Neurosci 14:3922-3933.

Lebkowski JS, Chaney S, Calos MP (1985) Simian virus 40 replication in adenovirus-transformed human cells antagonizes gene expression. Nature 317:169-171.

Leonard JP, Salpeter MM (1979) Agonist-induced myopathy at the neuromuscular junction is mediated by calcium. J Cell Biol 82:811-819.

Leonard JP, Salpeter MM (1982) Calcium-mediated myopathy at neuromuscular junctions of normal and dystrophic muscle. Exp Neurol 46:121-138.

Levitt TA, Salpeter MM (1981) Denervated endplates have dual population of junctional acetylcholine receptors. Nature 291:239-241.

Liu E, Hamill OP, Salpeter MM (1994) Mouse muscle $\epsilon$ - and $\gamma$ containing acetylcholine receptors expressed in Xenopus laevis oocytes do not differ in their degradation half-lives. Neurosci Lett 174:77-80.

Michler A, Sakmann B (1980) Receptor stability and channel conversion in the subsynaptic membrane of the developing mammalian neuromuscular junction. Dev Biol 80:1-17.

Miles K, Greengard P, Huganir RL (1989) Calcitonin gene-related peptide regulates phosphorylation of the nicotinic acetylcholine receptor in rat myotubes. Neuron 2:1517-1524.

Mishina M, Takai T, Imoto K, Noda M, Takahashi T, Numa S, Methfessel C, Sakmann B (1986) Molecular distinction between fetal and adult forms of muscle acetylcholine receptor. Nature 321:406-411.

Missias AC, Chu GC, Klocke BJ, Sanes JR, Merlie JP (1996) Maturation of the acetylcholine receptor in skeletal muscle: regulation of the AChR $\gamma$-to- $\epsilon$ switch. Dev Biol 179:223-238.

Naranjo D, Brehm P (1993) Modal shifts in acetylcholine receptor channel gating confer subunit-dependent desensitization. Science 260:1811-1814.

Nelson S, Shelton GD, Lei S, Lindstrom JM, Conti-Tronconi BM (1992) Epitope mapping of monoclonal antibodies to Torpedo acetylcholine receptor $\gamma$ subunits which recognize the $\epsilon$ subunit in mammalian muscle acetylcholine receptor. J Neuroimmunol 36:13-27.

O’Malley JP, Rubin LL, Salpeter MM (1993) Two populations of AChR in rat myotubes have different degradation rates and responses to cAMP. Exp Cell Res 208:44-47.

O’Malley JP, Greenberg I, Salpeter MM (1996) The production of longterm rat muscle cell cultures on a Matrigel substrate and the removal of fibroblast contamination by collagenase. Methods Cell Sci 18:19-655-661.

O’Malley JP, Moore CT, Salpeter MM (1997) Stabilization of AChRs by exogenous ATP and its reversal by cAMP and calcium. J Cell Biol 138:159-165.

Pinset C, Mulle C, Benoit P, Changeaux J, Chelly J, Gros F, Montarras D (1991) Functional adult acetylcholine receptor develops independently of motor innervation in Sol 8 mouse muscle cell line. EMBO J 10:2411-2418.

Reiness CG, Weinberg CB (1981) Metabolic stabilization of acetylcholine receptors at newly formed neuromuscular junctions in rat. Dev Biol 84:247-254.

Rich MM, Lichtman JW (1989) In vivo visualization of pre- and postsynaptic changes during synapse elimination in reinnervated mouse muscle. J Neurosci 9:1781-1805.

Rubin LL (1985) Increases in muscle $\mathrm{Ca}^{2+}$ mediate changes in acetylcholinesterase and acetylcholine receptors caused by muscle contraction. Proc Natl Acad Sci USA 82:7121-7125.

Sakmann B, Brenner HR (1978) Change in synaptic channel gating during neuromuscular development. Nature 276:401-402.

Salpeter MM, Loring RH (1985) Nicotinic acetylcholine receptors in vertebrate muscle: properties distribution and neural control. Prog Neurobiol 25:297-325.

Salpeter MM, Cooper DL, Levitt-Gilmour T (1986) Degradation rates of acetylcholine receptors can be modified after they are inserted into the postjunctional plasma membrane of the vertebrate neuromuscular junction. J Cell Biol 103:1399-1403.

Salpeter MM, Andreose J, O'Malley JP, Xu R, Fumagalli G, Lomo T (1993) Degradation of acetylcholine receptors at vertebrate neuromuscular junctions. In: Myasthenia gravis and related disorders: experimental and clinical aspects (Penn AS, Richman DP, Ruff RL, Lennon VA, eds), pp 155-164. New York: New York Academy of Sciences.

Shepherd D, Brehm P (1994) Adult forms of nicotinic acetylcholine receptors are expressed in the absence of nerve during differentiation of a mouse skeletal muscle cell line. Dev Biol 161:549-557.

Shyng SL, Salpeter MM (1989) Degradation rate of acetylcholine receptors inserted into denervated vertebrate neuromuscular junctions. J Cell Biol 108:647-651.

Shyng SL, Salpeter MM (1990) Effect of reinnervation on the degradation rate of junctional acetylcholine receptor synthesized in denervated skeletal muscle. J Neurosci 10:3905-3915.

Shyng SL, Xu R, Salpeter MM (1991) Cyclic AMP stabilizes the degradation of original junctional acetylcholine receptors in denervated muscle. Neuron 6:469-475.

Siegelbaum SA, Trautmann A, Koenig J (1984) Single acetylcholineactivated channel currents in developing muscle cells. Dev Biol 104:366-379.

Silinski EM (1975) On the association between transmitter secretion and the release of adenine nucleotides from mammalian motor nerve terminals. J Physiol (Lond) 247:145-162.

Stanley EF, Drachman DB (1981) Denervation accelerates the degradation of junctional acetylcholine receptors. Exp Neurol 73:390-396.

Steinbach IH, Merlie J, Heinemann S, Bloch R (1979) Degradation of the junctional and extrajunctional acetylcholine receptor by developing rat skeletal muscle. Proc Natl Acad Sci USA 76:3547-3551.

Stiles JR, Salpeter MM (1997) Absence of nerve-dependent conversion of rapidly degrading to stable acetylcholine receptors at adult innervated endplates. Neuroscience, in press.

Tzartos S, Langeberg L, Hochschwender S, Swanson LW, Lindstrom J (1986) Characteristics of monoclonal antibodies to denatured Torpedo and to native calf acetylcholine receptors: species, subunit, and region specificity. J Neuroimmunol 10:235-253.

Vernino S, Rogers M, Radcliffe KA, Dani JA (1994) Quantitative measurement of calcium flux through muscle and neuronal nicotinic acetylcholine receptors. J Neurosci 14:5514-24.

Vicini S, Schuetze AM (1985) Gating properties of acetylcholine receptors at developing rat endplates. J Neurosci 5:2212-2224.

Wigler M, Sweet R, Sim GK, Wold B, Pellicer A, Lacy E, Maniatis T, Silverstein S, Axel R (1979) Transformation of mammalian cells with genes from prokaryotes and eukaryotes. Cell 17:777-785.

Witzemann V, Brenner HP, Sakmann B (1991) Neural factors regulate AChR subunit mRNAs at rat neuromuscular synapses. J Cell Biol 114:125-141.

Xu R, Salpeter MM (1995) Protein kinase A regulates the degradation rate of Rs acetylcholine receptors. J Cell Physiol 165:30-39.

Xu R, Salpeter MM (1997) Acetylcholine receptors in innervated muscles of dystrophic mdx mice degrade as after denervation. J Neurosci 17:8194-8200. 